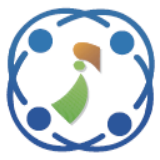

\title{
Corpus Linguistic Rules Based Review Sentence Selection for Opinion Targets Extraction and Opinion Orientation: A Distant Supervision Approach
}

\author{
Chiranjeevi Pandi ${ }^{1 *}$ \\ Teja Santosh Dandibhotla² \\ Vishnu Vardhan Bulusu ${ }^{3}$ \\ ${ }^{I}$ Department of Computer Science and Engineering, ACE Engineering College, Hyderabad, India \\ ${ }^{2}$ Department of Information Technology, \\ Sreenidhi Institute of Science and Technology, Ghatkesar, Hyderabad, India \\ ${ }^{3}$ Department of Computer Science and Engineering, \\ Jawaharlal Nehru Technological University Hyderabad College of Engineering Manthani, Manthani, India \\ * Corresponding author’s Email: chiruanurag@gmail.com
}

\begin{abstract}
Opinion Target Extraction (OTE) identifies opinionated aspects in review sentences with the goal of achieving Aspect Based Opinion Mining (ABOM). The available review corpuses and datasets for this task contain a large number of opinionated sentences from wider class of products from different e-Commerce applications. This raised the need for constructing the dataset from these corpora for OTE in a new way called distant supervision. This is by selecting the review sentences based on the learned opinionated relations among the terms in the sentence. The obtained review sentences are then labelled and thus dataset is constructed. The attention model based review sentence selection that uses distant supervision has ignored the review sentences that do not contain opinionated aspect terms and treated them as noise. This has reduced the precision in terms of number of extracted aspects. In order to improve the precision of extracted aspect terms, the attention based review sentence selection is replaced with the learned corpus linguistic rules based review sentence selection. This takes into account the non opinionated aspects available sentences and these aspects are treated as neutral opinion targets. Finally, the extracted aspect terms are analyzed for opinion orientations. It is shown that the approach of corpus linguistics rules based review sentence selection outperforms the attention model based review sentence selection with distant supervision.
\end{abstract}

Keywords: Aspect term, Dataset construction, Distant supervision, Sentence selection, Corpus linguistic rules, Neutral opinionated aspects, Aspects labelling, Opinion orientation.

\section{Introduction}

Sentiment analysis is increasingly viewed as a vital task both from an academic and a commercial standpoint. The majority of current approaches, however, attempt to detect the overall polarity of a sentence or text span regardless of the entities mentioned and their aspects. By contrast, the aspect based opinion mining (ABOM) identifies aspects of given target entities and the sentiment expressed towards each aspect. The first task as mentioned earlier in ABOM is OTE and the second task is to determine the opinion orientation of the extracted aspects.
The existing machine learning approaches for OTE are classified into supervised and unsupervised. Each class comes with many benefits and shortcomings. Supervised machine learning for OTE leads to high performance on unseen data. Supervised OTE depends on the manually labelled datasets and corpuses [1]. Unsupervised OTE utilize largely available public domain review corpora. These corpora cover large number of opinionated sentences from wider class of products from different e-Commerce applications. However, unsupervised OTE systems have less performance when compared with the supervised systems [2]. This raised the need for constructing the dataset from these corpora for OTE in a new way called distant supervision. This is by selecting the review 
sentences based on the learned opinionated relations among the terms in the sentence. The obtained review sentences are then labelled and thus dataset is constructed.

The attention model based review sentence selection that uses distant supervision has ignored the review sentences that do not contain opinionated aspect terms and treated them as noise. This has reduced the precision in terms of number of extracted aspects. In order to improve the precision of extracted aspect terms, the attention based review sentence selection is replaced with the learned corpus linguistic rules based review sentence selection. This takes into account the non opinionated aspects available sentences and these aspects are treated as neutral opinion targets.

The aspects are extracted by learning the Conditional Random Field (CRF) model on the selected sentences and testing the model with the separated test review sentences. The terms whose training class label and test class label are both with the same aspect specified label then those terms are treated as aspects. Finally, the extracted aspect terms are analyzed for opinion orientations.

This paper is organised as follows: related work is described in section 2, the proposed model is explained in Section 3, the experimental procedure of the proposed approach and the results are discussed in Section 4 and finally the conclusions and the scope for future work are presented in Section 5.

\section{Related work}

Research in the area of both supervised and unsupervised OTE has increased since the first SemEval ABOM task which had taken place in 2014. Participants who worked on supervised OTE [3 - 5] used the provided human labelled datasets in order to extract linguistic features. These features are very similar to those used in traditional Name Entity Recognition (NER) systems [6]. However, these works on supervised OTE achieved less accuracy on aspect term extraction subtask. Moreover, participants exploited external sources such as WordNet and word clusters like Brown corpus based clusters [7]. Finally, they exploited gazetteers [8] and word embeddings [9]. The extracted features are used to train a classifier such as Conditional Random Fields (CRF) or Support Vector Machine (SVM). These research works mainly focused on the task of summarization.

Very less works concentrated on OTE. The works of [10] and [11] investigate sentence clustering in order to acquire new similar sentences to improve their model. The former work focuses on aspect identification while the latter on multi-aspects review summarization. The work of [12] explores the use of a community-leader detection problem with sentence selection in order to build better opinion summarization, where communities consist of a cluster of sentences towards the same aspect of an entity. The work of [13] performed OTE using sentence selection with distant supervision by using attention model. This is the first work on OTE with distant supervision. The attention model based review sentence selection has ignored the review sentences that do not contain opinionated aspect terms and treated them as noise.

The semantic orientation of texts is an age old classical work for more than five decades. Osgood et al. identified [14] several pairs of bipolar adjectives that greatly influence the shift in the orientation of the opinion words. Hatzivassiloglou and McKeown attempted [15] to predict the orientation of the opinion words by analyzing the pairs of adjectives bounded by conjunctions. Turney and Littmann approached [16] the problem by using a seed set to bootstrap the process of opinion word identification for the first time. Once the opinion words are identified, Pointwise Mutual Information (PMI) was calculated on the identified opinion word and the term in the seed set. The work [15] on determining the orientation of the terms is concentrated on pairs of adjectives bounded by conjunctions. The researchers considered only 657/679 documents (labelled Positive/Negative) in which the adjectives bound by conjunctions are available from the Wall Street Journal (WSJ) corpus.

Kamps et al. focused [17] on the relations between the words defined in the WordNet. They calculated the relative distance from the two seed terms to the identified opinion word to determine the orientation of the opinion word. The work of solving the ambiguity of the terms that appear in both the Positive and Negative categories was never concentrated [17]. They removed those terms from the sets and experimented on the reduced sets. The number of considered terms after removing the ambiguous entries is $1614 / 1982$. They restricted the adjectives in the analysis to 663 from the total 3596 terms of Turney and Littmann as used in [16]. This is because the synonymy relation graph of WordNet evaluates only those adjectives that are in the path of the graph bounded with the seed terms at the ends of the graph. Recently, Emiel van Miltenburg calculated [24] the distance between two adjectives by obtaining derivationally related forms of the adjectives. These derivationally related forms are associated with the adjective lemmas. 
The attention model based review sentence selection has ignored the review sentences that do not contain opinionated aspect terms and treated them as noise. This has reduced the precision in terms of number of extracted aspects. In order to improve the precision of extracted aspect terms, the attention based review sentence selection is to be replaced with the learned corpus linguistic rules based review sentence selection. This takes into account the non opinionated aspects available sentences and these aspects are treated as neutral opinion targets.

All the above works on SO never concentrated on assigning weights to the opinion words based on the context based sense. This provides a better way of evaluating the semantic orientation of the opinion words. An alternate way of determining the semantic orientation of the terms in the manner is required. In addition to this, the distance measure is to be modified in such a way that the relative distance which is calculated to determine the semantic orientation uses the Sentiwordnet [18] scores.

\section{Dataset construction via corpus linguistic rules based review sentence selection for opinion target extraction}

Specified with an online review, it often occurs that only the part of the sentence expresses the opinion on the entity. Also some of the review sentences do not contain the opinionated view on the entity. The example for such review is, "I bought this camera from Amazon three weeks ago". The opinions for such sentences are analyzed as having neutral valence. These kinds of sentences are also considered for dataset construction towards OTE.

The hypothesis framed in this work is that sentence selection matters in constructing the dataset for improved OTE in terms of precision. In other words, training a classifier on the selected reviews for dataset using learned linguistic patterns from the corpuses leading to a better OTE performance.

The proposed model for OTE with distant supervision is depicted in Fig. 1 below. The model starts with collecting the review corpora and datasets on a particular product from which the OTE specific review sentences are to be selected. Then the manufacturer listed aspects in the e-commerce website are also collected for this task.

Now these listed aspects are applied against the reviews by using the learned corpus linguistic patterns to select the opinionated sentences from the reviews. These corpus linguistic patterns are represented in the form of conjunctive rules on the
Part-of-Speech (PoS) tagged terms and the dependency relations among these terms.

The selected review sentences from the application of the linguistic rules are constructed as a new dataset for OTE. Further, Conditional Random Field (CRF) based sequential term labelling of the sentences is carried out. Furthermore, CRF based training of the sentences is carried out on the training set to learn the classifier.

Then the test set is applied against the learned CRF model to identify and extract the opinion targets or aspects of the product. This completes the OTE task.

In order to perform ABOM, the extracted aspects are further analyzed for opinions by identifying the opinion words using the standard opinion lexicon and determining the semantic orientation of the opinion words by using the corresponding sentiwordnet scores.

\subsection{Review sentence selection using learned linguistic rules based on PoS terms and typed dependency relations}

The review sentence selection approach adopts the rule-based strategy which is quite natural that are learned from well-defined relations. For example, in the opinion sentence, "Canon G3 takes great pictures", the adjective 'great' is parsed as directly depending on the noun 'pictures' through mod dependency relation. So, to perform review sentence selection, the reviews are tagged by the PoS tagger first and the dependency relations among these tagged terms are obtained next. The pipeline diagram for the aforementioned task is depicted in Fig. 2 below.

The stop words that are used across all the reviews are removed as they cannot actually infer any meaning. The stop words are compiled from the reviews itself. This compilation is carried out by sorting the terms in the decreasing order of collection frequency and thereby hand-filtering those terms for their semantic content relative to the reviews domain.

Then in order to select aspect specific review sentences from the corpuses, PoS tagging is performed. PoS tagging is carried out to relate the word with the corresponding word class tag. It helps in identifying the actual product features in further semantic analysis during feature extraction. Stanford Part-of-Speech Tagger [19] framework is used to tag the words.

Further, the review sentences with PoS tagged words are parsed for term dependencies among them. 


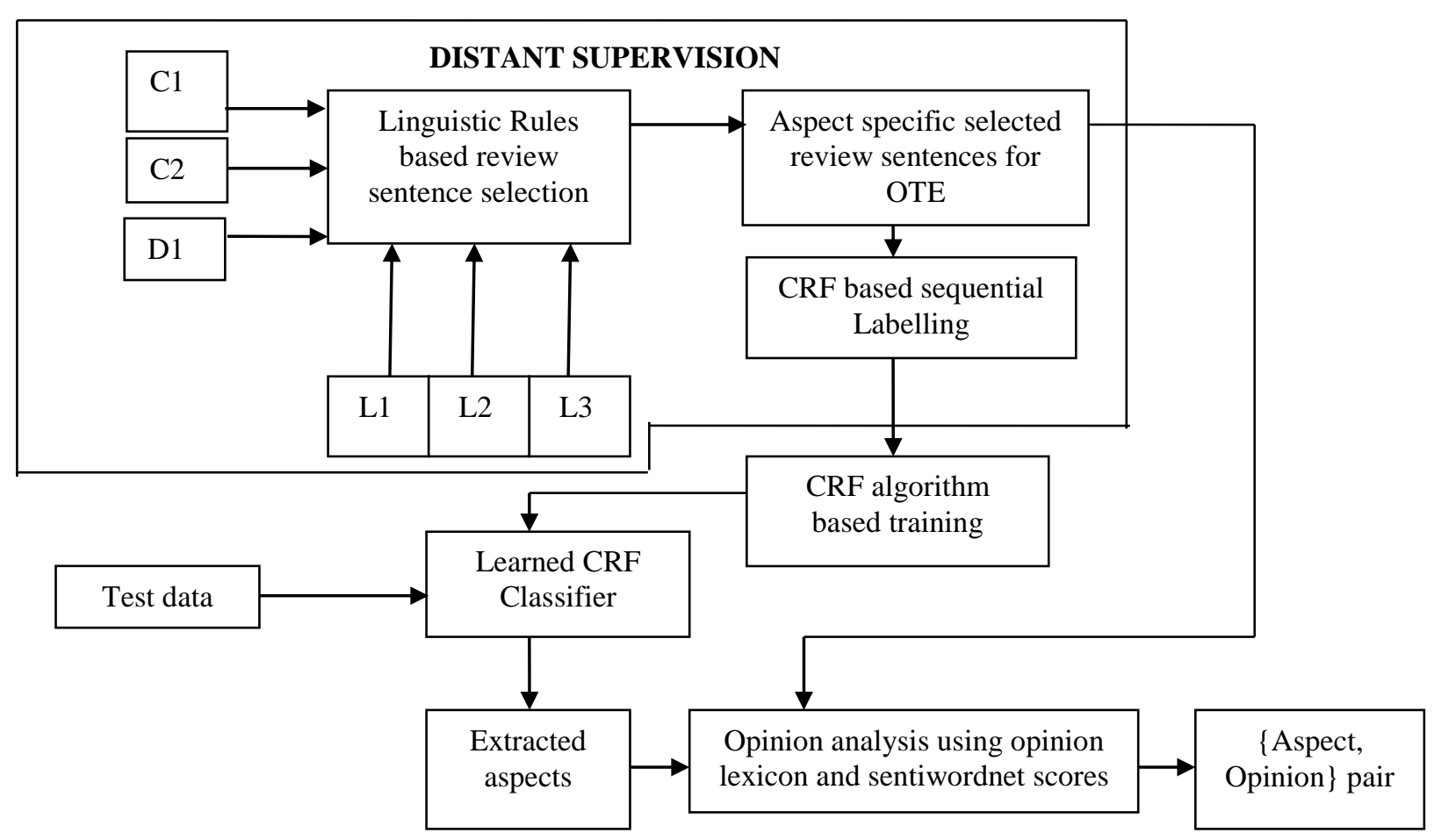

Figure. 1 Proposed model

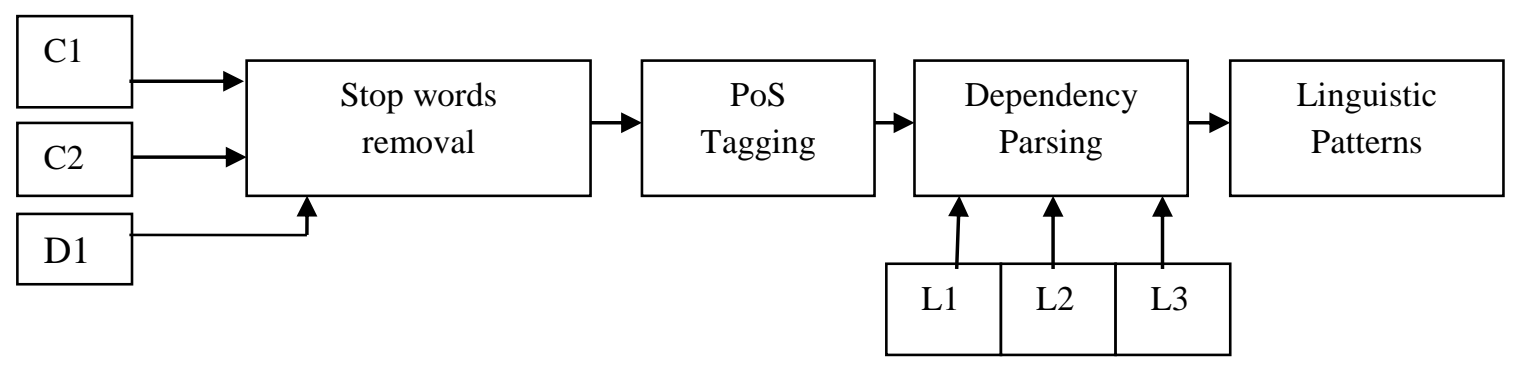

Figure. 2 Pipeline for learning linguistic patterns from review sentences

These term dependencies provide a simple description of the grammatical relationships in a sentence that can easily be understood by the machine with the manufacturer list of product aspects towards unambiguous sentence selection. For the considered review sentence "I love Apple iPhone 6s Plus and will recommend my friend also" the typed dependency parsed sentence is shown in below Fig. 3.

Now the relations among the terms in the typed dependency reviews are carefully analyzed for aspect specific sentences with the help of manufacturer list of aspects. The rule antecedent is the linguistic patterns of opinionated aspects occurring among the review sentences. The rule consequent is select_sentence(S) which is a function that chooses the sentence when the sentence covers opinionated aspects. The learned linguistic rules based on the PoS tags and typed dependencies are given below.

Rule 1: term_NN term_JJ then select_sentence(S)

Rule 2: term_NN term_JJ and term_JJ nsubj term_NN then select_sentence(S)

Rule 3: term_JJ term_NN then select_sentence(S)

Rule 4: term_JJ term_NN and term_NN dobj term_JJ then select_sentence(S)

Rule 5: term_JJ term_NN and term_NN amod term_JJ then select_sentence(S)

Rule6:term1_NN term2_NN term3_JJ and term1_NN compound term2_NN then select_sentence(S)

Rule7:term1_NN term2_NN term3_JJ and term1_NN conj term2_NN then select_sentence(S) 


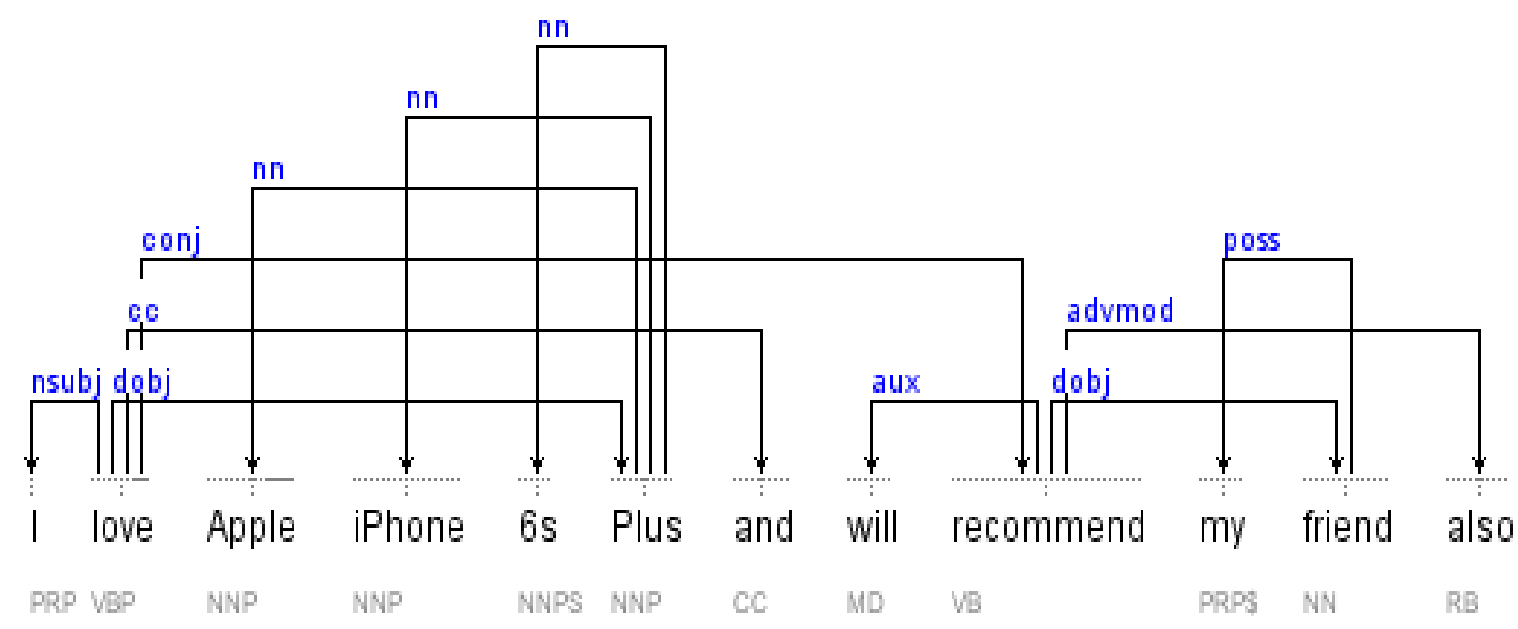

Figure. 3 Type dependencies in the review sentence

Table 1. Some of the selected review sentences based on the learned linguistic rules

\begin{tabular}{|c|c|c|}
\hline $\begin{array}{c}\text { Linguistic } \\
\text { Rule }\end{array}$ & $\begin{array}{c}\text { Selected Review } \\
\text { Sentence }\end{array}$ & $\begin{array}{c}\text { Candidate } \\
\text { Aspects for } \\
\text { extraction }\end{array}$ \\
\hline Rule 4 & I love this camera & camera \\
\hline Rule 6 & $\begin{array}{c}\text { The battery life is } \\
\text { excellent }\end{array}$ & battery life \\
\hline Rule7 & $\begin{array}{c}\text { The RAM and } \\
\text { performance of are } \\
\text { terrible }\end{array}$ & $\begin{array}{c}\text { RAM, } \\
\text { performance }\end{array}$ \\
\hline Rule 10 & $\begin{array}{c}\text { I am using this } \\
\text { earphones since six } \\
\text { months }\end{array}$ & earphones \\
\hline
\end{tabular}

Rule 8: term1_NN term2_RB term3_JJ and term3_JJ nsubj term1_NN and term3_JJ advmod term2_RB then select_sentence $(\mathrm{S})$

Rule 9: term_VB term_NN and term_VB dobj term_NN then select_sentence $(\mathrm{S})$

Rule 10: term_NN/term_NNS then select_sentence( $\mathrm{S})$

The notation "term_PoS tag" is the term in the linguistic pattern and the words specified in italics are the typed dependency grammatical relations. The subset of the selected review sentences from the corpuses are constructed as a new dataset for extracting product aspects. Some of the selected review sentences based on the learned linguistic rules are tabulated in Table 1 given above.

\subsection{Sentences dataset labelling and training using CRF sequential modelling}

The review sentences in the newly constructed dataset is labelled sequentially with ' $A$ ' for nouns and noun phrases in order to extract them as aspects, ' $\mathrm{OW}$ ' for adjective terms in order to extract them as opinion words and ' $\mathrm{O}$ ' for all other PoS tagged terms. The sequential labelling method used is Conditional Random Fields.

Given the sequence of terms in the review sentence, a list of features for each term is encoded for CRF training. These features are as follows:

1. Review Term: This indicates which word type is the actual instance to be labelled.

2. PoS tag: The PoS tag of the word.

3. Class label: The labels are A for aspect terms (nouns), OW for adjectives and $\mathrm{O}$ for other PoS terms.

Following is the encoding approach of the review terms in the sequence for the review "keyboard and sound are awful".

\begin{tabular}{|c|c|c|}
\hline Review Term & PoS tag & Class label \\
\hline ---------------- & --------- & ------- \\
\hline keyboard & NN & $\mathrm{A}$ \\
\hline and & $\mathrm{CC}$ & $\mathrm{O}$ \\
\hline sound & NN & A \\
\hline are & VBP & $\mathrm{O}$ \\
\hline awful & $\mathrm{JJ}$ & OW \\
\hline
\end{tabular}

The training of the labelled review sentences is carried out using supervised CRF classifier. Conditional Random Fields (CRF) [22] is one of the popular probabilistic graphical models for building probabilistic models to divide and label sequences of data. It is a discriminative model. More generally, a CRF is a log-linear model that defines a probability distribution over sequences of data given a particular observation sequence.

In terms of set of observations $X$ and a set of label sequences $\mathrm{Y}$, the CRF probabilistic graphical model ' $G$ ' with the collection of vertices ' $\mathrm{V}$ ' indicating observations and labels and edges ' $E$ ' indicating the mapping (dependencies) among 
observations and labels. These edges help in learning the patterns among the observations and labels towards learning the decision boundary.

The posterior conditional probability in learning label sequences is given by;

$$
p(Y \mid X)=\frac{1}{Z(X)} \mathrm{X} \exp \left\{\sum_{m=1}^{M} \lambda m f m(y n, y n-1, x n)\right\}
$$

Here $\mathrm{Z}(\mathrm{X})$ is the instance specific partition function. This is given by;

$$
Z(X)=\sum_{y} \exp \left\{\sum_{m=1}^{M} \lambda m f m(y n, y n-1, x n)\right\}
$$

where $\mathrm{m}$ is the feature subscript, $\mathrm{y}_{\mathrm{n}}$ is the current term label, $\mathrm{y}_{\mathrm{n}-1}$ is the previous term label and $\mathrm{x}_{\mathrm{n}}$ is the current term. "Lambda" is the probability distribution parameter on the observations in the entire corpus and $f_{m}$ are feature functions. This means that the term label prediction from the test sentence is based on the current term and previous term trained labels, the term probability distribution in the entire reviews collection of that product and, the word with its PoS tag representation.

\subsection{Aspect extraction from labelled sentences using learned CRF classifier}

The test review sentences are applied against the learned CRF classifier. A new column is generated which is provided by the classifier. This column data is the classifier provided class label. In order to extract aspect terms, the terms whose training class label and test class label are both with the label ' $A$ ' are extracted and counted. This completes the aspect extraction process.

\subsection{Opinion analysis using opinion lexicon and sentiwordnet scores}

The adjectives that are present either before or after the identified aspects are extracted. These adjectives are analysed for opinions and orientations in the following steps.

(i) A standard opinion lexicon in which two sets of adjectives are present is considered as input for bootstrapping. These sets represent two categories namely Positive and Negative. Two seed terms 'good' and 'bad' represent the two categories are taken into consideration. (ii) The sizes of the Positive and Negative adjective sets are increased by adding the synonyms and antonyms of the adjectives using WordNet [23].

(iii) The increased sizes of Positive and Negative adjective sets are used to compare with the obtained adjectives from the dataset. Once the dataset adjectives are matched with the opinion lexicon adjectives, then the dataset adjectives are considered as opinion words. This completes the identification of opinion words from the dataset.

(iv) The opinion word and the seed terms are assigned with the numerical scores available under adjective category from Sentiwordnet. This is carried out by finding the contextual clues surrounding the opinion word [21]. These contextual clues will help to disambiguate the sense of the opinion word. The contextual clues are finalized based on the typed dependency grammatical relations.

(v) The distance between the opinion word and the seed term and the distance between the seed terms is calculated as given below.

$\operatorname{distance}\left(\mathrm{w}_{\mathrm{i}}, \mathrm{w}_{\mathrm{j}}\right)$

$=\operatorname{sentiwordnetscore}\left(\mathrm{w}_{\mathrm{i}}\right)-\operatorname{sentiwordnetscore}\left(\mathrm{w}_{\mathrm{j}}\right)$

where $\mathrm{w}_{\mathrm{i}}$ is either the opinion word or the seed term and $\mathrm{w}_{\mathrm{j}}$ is the seed term. The distance measure is modified as the application of distance is carried on non-hierarchical semantic network [20] i.e., on adjectives.

(vi) The semantic orientation ( $\mathrm{SO}$ ) of the opinion word is determined as given below.

$\mathrm{SO}($ opinion word $)=$ distance $($ opinion word, bad $)-$ distance(opinion word, good)

distance (good, bad)

(vii) The opinion word is deemed to be positive when the orientation measurement is greater than zero, and negative otherwise.

Finally the extracted aspects and the corresponding opinions are generated as a pair. This completes the ABOM task.

\section{Experiments and results discussion}

The datasets used for the task of review sentence selection for opinion targets extraction and opinion orientation with distant supervision are the 
Table 2. Dataset details

\begin{tabular}{|c|c|}
\hline Document attributes & Values \\
\hline Number of review documents & 67458 \\
\hline Minimum sentences per review & 2 \\
\hline Maximum sentences per review & 43 \\
\hline $\begin{array}{c}\text { Average number of reviews written by } \\
\text { customers }\end{array}$ & 3.78 \\
\hline $\begin{array}{c}\text { Average number of reviews written on the } \\
\text { product }\end{array}$ & 28.47 \\
\hline
\end{tabular}

Table 3. Constructed dataset details after rules based review sentences selection

\begin{tabular}{|c|c|c|}
\hline $\begin{array}{c}\text { Product } \\
\text { Category }\end{array}$ & $\begin{array}{c}\text { No. of selected } \\
\text { review sentences }\end{array}$ & $\begin{array}{c}\text { No. of } \\
\text { products }\end{array}$ \\
\hline $\begin{array}{c}\text { E- } \\
\text { commerce } \\
\text { application }\end{array}$ & Amazon & Amazon \\
\hline $\begin{array}{c}\text { GPS } \\
\text { Devices }\end{array}$ & 11441 & 100 \\
\hline Tablets & 12645 & 100 \\
\hline Laptops & 5683 & 100 \\
\hline $\begin{array}{c}\text { Smart } \\
\text { phones }\end{array}$ & 9082 & 100 \\
\hline Cameras & 4587 & 100 \\
\hline
\end{tabular}

Table 4. Number and percentage of neutral review sentences

\begin{tabular}{|c|c|c|}
\hline $\begin{array}{c}\text { Product } \\
\text { Category }\end{array}$ & $\begin{array}{c}\text { No. of neutral } \\
\text { review sentences }\end{array}$ & $\begin{array}{c}\text { Percentage of } \\
\text { neutral review } \\
\text { sentences (\%) }\end{array}$ \\
\hline GPS Devices & 1716 & 15.5 \\
\hline Tablets & 2276 & 18.4 \\
\hline Laptops & 909 & 15.7 \\
\hline $\begin{array}{c}\text { Smart } \\
\text { phones }\end{array}$ & 1725 & 22.1 \\
\hline Cameras & 779 & 22.4 \\
\hline
\end{tabular}

collection of five categories of product reviews from Amazon. GPS devices, Tablets, Laptops, Smart phones and cameras are the product categories for which the reviews are considered for analysis. In each product category, 100 products are considered from the E-commerce application. Table 2 presents the details of the datasets used for this experiment.

The pre-processing of data is carried out by removing stop words and non English words. PoS tagging is performed on the obtained set of words.
Table 5. Accuracy of the CRF classifier on the extracted aspects

\begin{tabular}{|c|c|c|c|}
\hline Category & Precision $(\boldsymbol{\%})$ & $\begin{array}{c}\text { Recall } \\
(\boldsymbol{\%})\end{array}$ & $\begin{array}{c}\text { F1-Score } \\
(\boldsymbol{\%})\end{array}$ \\
\hline $\begin{array}{c}\text { GPS } \\
\text { Devices }\end{array}$ & 65.8 & 70.5 & 68.1 \\
\hline Tablets & 68.7 & 74.3 & 71.4 \\
\hline Laptops & 66 & 72.9 & 69.2 \\
\hline $\begin{array}{c}\text { Smart } \\
\text { phones }\end{array}$ & 69.3 & 75.8 & 72.4 \\
\hline Cameras & 67.3 & 79.1 & 72.7 \\
\hline
\end{tabular}

The nouns, adjectives, verbs and adverbs present in the review sentences are analyzed for aspect specific opinionated patterns towards OTE specific dataset construction. The constructed dataset details after review sentences selection from the five product categories are tabulated in the Table 3.

The number and the percentage of review sentences that do not contain the opinionated view on the entity from the five product categories are tabulated in Table 4.

The statistics from the above table provide the information that less than 25 percent of the reviews contain neutral review sentences across all the five product categories.

The precision, recall and F1-scores on the extracted aspects by using CRF classifier on the five product categories is tabulated in Table 5 above.

The results from the above table specify that the precision acquired using corpus linguistics rules based review sentence selection is $67.42 \%$. This has a significant increase of $17 \%$ when compared with the precision of attention model based review sentence selection which is $50.33 \%$. This shows that the approach of corpus linguistics rules based review sentence selection outperformed the attention model based review sentence selection with distant supervision. This is because the neutral review sentences are also selected for aspect extraction which were treated as noise in attention model based review sentence selection work.

The comparative analysis of the proposed approach to that of attention model is presented in Fig. 4 below.

The improved $\mathrm{Hu}$ and Liu opinion lexicon dataset was used in this experiment to extract the opinions from the reviews. The opinion lexicon is improved by adding 2290 unique positive words and 4800 unique negative words from SentiWordNet. Table 6 presents the details of the dataset used for this experiment. Fifteen reviews from the five 


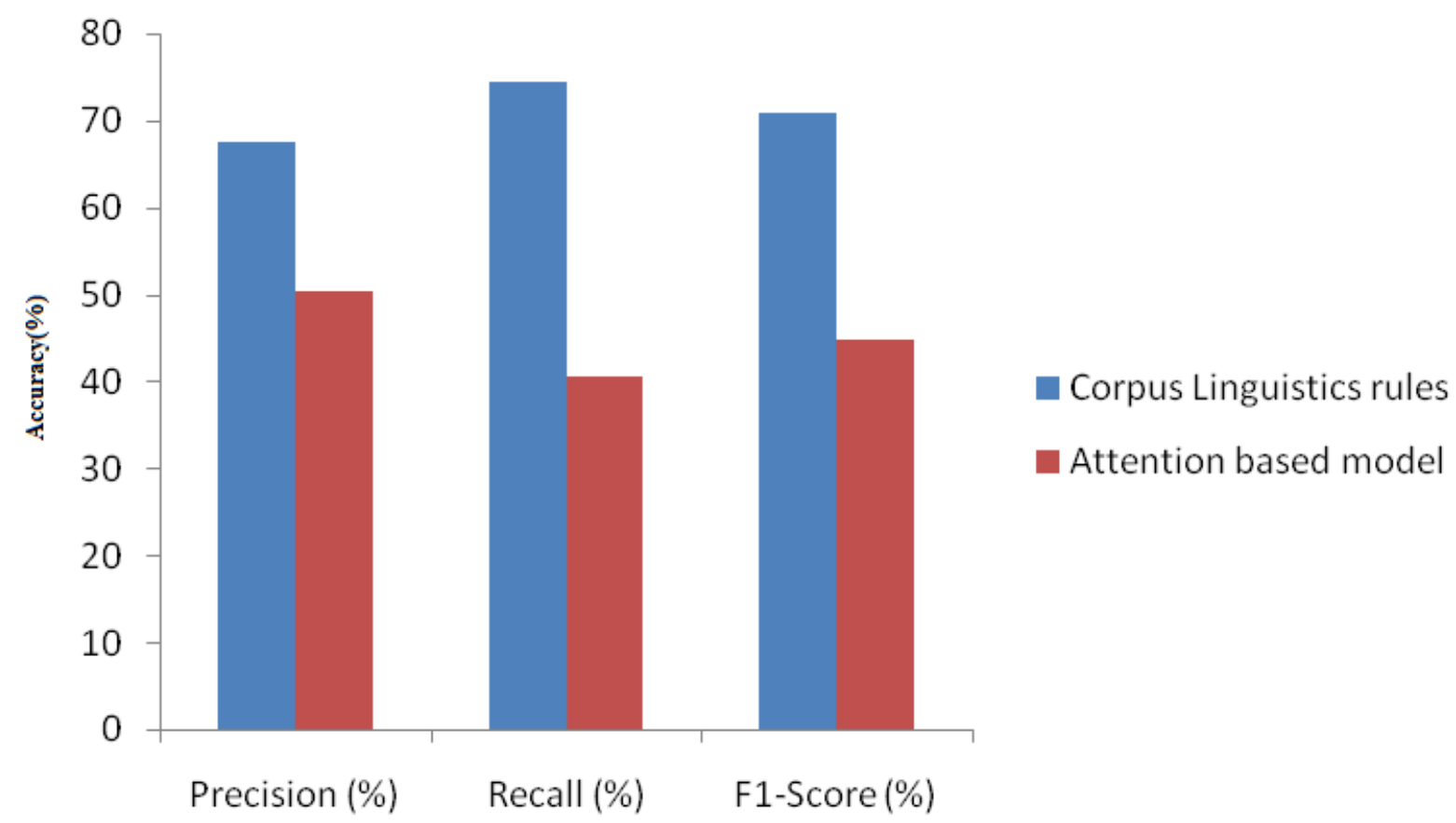

Figure. 4 Comparative analysis

Table 6. Opinion lexicon details

\begin{tabular}{|l|c|}
\hline Opinion word attributes & Values \\
\hline Number of positive words & 4294 \\
\hline Number of negative words & 9582 \\
\hline
\end{tabular}

datasets from these five product categories were taken to extract the opinions and determine their orientations. The reviews are given below.

Apple iphone 6s plus

1. Siri is awesome do most of the work smoothly.

2. Very happy to get an iPhone 6 s plus.

3. Really good phone and its my first iPhone. Great screen size and good battery life.

\section{Letstrack Bike Tracing GPS Device}

1. Good work guys. The way you guys install it, is good as it is always hidden. Now I track my bike wherever it is and I really like the parking alert feature.

2. This is best for tracking. I am using this device. This is very good for bike GPS tracking.

3. Perfect.

\section{Micromax Canvas Tab P70221}

1. Good budget tablet in 5000 range.

2. Camera is not good.

3. Amazing Tablet. wonderful wifi+3G.

\section{Lenovo Core i5 $7^{\text {th }}$ Gen Laptop}

1. Awesome laptop. Looks are good

2. laptop has attractive design it give much more performance than i hoped.

3. Nice product and it's able to run GTA V without lag and in high graphic.

Nikon B700 Point and Shoot Camera

1. Nice .... camera sensor is small but good.

2. This is the best camera in this price range.

3. Nice product with pouch and $8 \mathrm{gb}$ card free.

After PoS tagging, the noun, adjective pairs found from the five datasets are as follows.

Iphone 6s plus: [(Siri, awesome), (iPhone 6s plus, happy), (phone, good), (screen size, great), (battery life, good)]

Letstrack Bike Tracing GPS Device: [(parking alert feature, good), (installation, good), (bike GPS tracking, good), (device, good)]

Micromax Canvas Tab P70221: [(budget, good), (camera, not good), (tablet, amazing), (wifi $+3 \mathrm{G}$, wonderful)]

Lenovo Core i5 $7^{\text {th }}$ Gen Laptop: [(laptop, awesome), (appearance, good), (design, attractive), (performance, better), (product, nice), (gaming, good), (graphics, high)] 


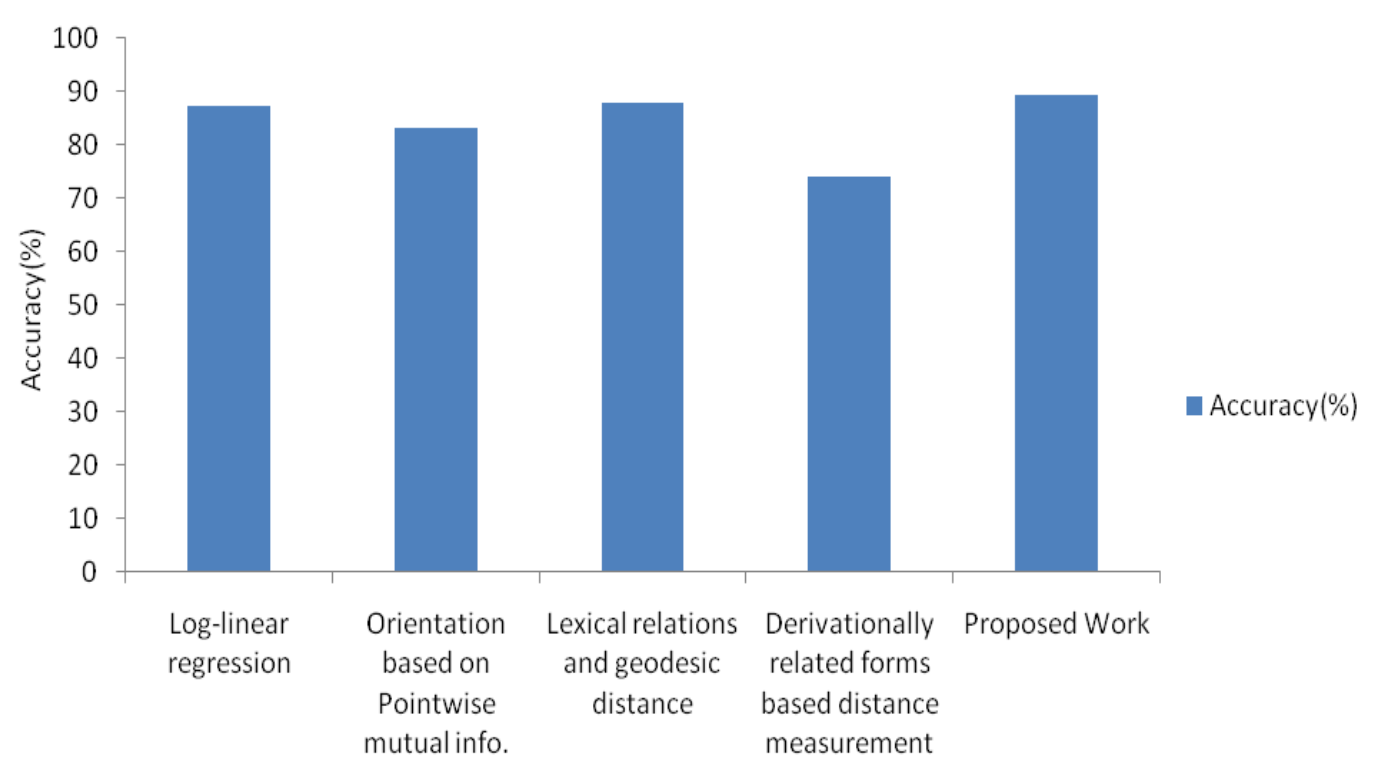

Figure 5. Accuracy of extracted opinions

Table 7. Accuracy (in \%) of extracted opinions

\begin{tabular}{|c|c|c|}
\hline $\begin{array}{c}\text { Opinion } \\
\text { Orientation } \\
\text { Method }\end{array}$ & $\begin{array}{c}\text { Pos./Neg. } \\
\text { Adjectives }\end{array}$ & Accuracy(\%) \\
\hline $\begin{array}{c}\text { Log-linear } \\
\text { regression [15] }\end{array}$ & $657 / 679$ & 87.38 \\
\hline $\begin{array}{c}\text { Orientation based } \\
\text { on Pointwise } \\
\text { mutual info. [16] }\end{array}$ & $1915 / 2291$ & 83.09 \\
\hline $\begin{array}{c}\text { Lexical relations } \\
\text { and geodesic } \\
\text { distance[17] }\end{array}$ & 663 of [15] & 88.05 \\
\hline $\begin{array}{c}\text { Derivationally } \\
\text { related forms } \\
\text { based distance } \\
\text { measurement [24] }\end{array}$ & $310 / 310$ & 74 \\
\hline Proposed Work & $2004 / 4782$ & 89.5 \\
\hline
\end{tabular}

Nikon B700 Point and Shoot Camera: [(product, nice), (camera sensor, small), (camera sensor, good), (camera, best), (price, good), (product, nice), (pouch, nice), (memory card, free)]

All the adjectives were compared with the opinion lexicon. All were identified. The identified adjectives were deemed as opinion words.

In order to determine the orientations of the opinion words, the senses of the opinion words were disambiguated by learning the context using typed dependencies [21] and WordNet gloss. The obtained sense is used in searching for the SentiWordNet score under adjectives category. The scores were substituted in SO formula. When the obtained value after SO calculation is greater than zero, then the opinion word is termed as positive, otherwise negative.

The evaluation on the orientation of the opinions using the proposed approach as compared with the baseline approaches is presented in table 7 below.

The results obtained in terms of accuracy with the published techniques are as shown in the Table 7, note that there are improvements in the orientation of the opinions in the work of measuring semantic orientation of adjectives using WordNet [23] when compared with log linear classifier based semantic orientation of adjectives [15] and PMI based semantic orientation of adjectives [16]. However, the accuracy of the proposed method when compared to the method of $[17,24]$ has increased in a significant manner. The comparative results are shown in Fig. 5.

\section{Conclusion}

The aspects extraction and their opinion orientation using learned corpus linguistic rules for review sentence selection with distant supervision were carried out successfully. The objective is to extract all kinds of opinionated aspects from review sentences. The dataset construction has started with applying corpus linguistic rules on the publicly available online reviews. Then the tokens of the constructed dataset are annotated with the specific labels for training the CRF classifier. The trained CRF classifier has been tested on the test dataset.

The precision acquired using the CRF classifier is $67.42 \%$. There was a significant increase of $17 \%$ when compared with the precision of attention model based review sentence selection which is $50.33 \%$. It has been observed from these 
experimental results that the accuracies of the proposed aspect extraction and opinion word orientation are better in terms of information retrieval measures when compared with the existing approaches.

The experiments carried out and the obtained results from this research validate the hypothesis framed in this work that sentence selection matters in constructing the dataset for improved OTE in terms of precision.

In future, these opinion words with their coexisted aspects are analyzed for entity reputation. Reputation analysis helps to discover overall sentiment on that entity. It also helps to determine from the reviews those mentions that may harm the brand name of the entity. This helps to handle the problem of customer churn in indefinite manner in a better way.

\section{References}

[1] S. Moghaddam and M. Ester, "Aspect-based opinion mining from online reviews", In: Proc. of SIGIR Conference, 2012.

[2] M. Pontiki, D. Galanis, H. Papageorgiou, I. Androutsopoulos, S. Manandhar, A.S. Mohammad, M. Al-Ayyoub, Y. Zhao, B. Qin, O. De Clercq, and V. Hoste, "SemEval-2016 task 5: Aspect based sentiment analysis", In: Proc. of the 10th International Workshop on Semantic Evaluation, pp. 19-30, 2016.

[3] Z. Toh and W. Wang, "Dlirec: Aspect term extraction and term polarity classification system", In: Proc. of the 8th International Workshop on Semantic Evaluation, pp. 235-240, 2014.

[4] Z. Toh and J. Su, "Nlangp: Supervised machine learning system for aspect category classification and opinion target extraction", In: Proc. of the 9th international workshop on semantic evaluation, pp. 496-501, 2015.

[5] M. Chernyshevich, "Ihs r\&d belarus: Crossdomain extraction of product features using crf", In: Proc. of the 8th International Workshop on Semantic Evaluation, pp. 309-313, 2014.

[6] M. Tkachenko and A. Simanovsky, "Named entity recognition: Exploring features", In: Proc. of 11th Conference on Natural Language Processing, pp. 118-127, 2012.

[7] J. Turian, L. Ratinov, and Y. Bengio, "Word representations: a simple and general method for semi-supervised learning", In: Proc. of the 48th Annual Meeting of the Association for Computational Linguistics, pp. 384-394, 2010.
[8] J. Kazama and K. Torisawa, "Inducing gazetteers for named entity recognition by largescale clustering of dependency relations", In: Proc. of ACL-08: HLT, pp.407-415, 2008.

[9] T. Mikolov, I. Sutskever, K. Chen, G.S. Corrado, and J. Dean, "Distributed representations of words and phrases and their compositionality", In: Proc. of the 27th Annual Conference on Neural Information Processing Systems, pp. 3111-3119, 2013.

[10] M. Hadano, K. Shimada, and T. Endo, "Aspect identification of sentiment sentences using a clustering algorithm", In: Proc. of 12th Conference of the Pacific Association for Computational Linguistics, pp.22-31, 2011.

[11] K. Shimada, R. Tadano, and T. Endo, "Multiaspects review summarization with objective information", In: Proc. of the 12th Conference of the Pacific Association for Computational Linguistics, pp.140-149, 2011.

[12] L. Zhu, S.Gao, S.J. Pan, H. Li, D. Deng, and C. Shahabi, "Graph-based informative-sentence selection for opinion summarization", In: Proc. of the 2013 IEEE/ACM International Conference on Advances in Social Networks Analysis and Mining, pp. 408-412, 2013.

[13] A. Giannakopoulos, D. Antognini, C. Musat, A. Hossmann, and M. Baeriswyl, "Dataset Construction via Attention for Aspect Term Extraction with Distant Supervision", In: Proc. of the 7th ICDM Workshop on Sentiment Elicitation from Natural Text for Information Retrieval and Extraction, 2017.

[14] C.E. Osgood, G.J. Suci, and P.H. Tannenbaum, "The measurement of meaning", University of Illinois Press, pp. 1-342, 1957.

[15] V. Hatzivassiloglou and K.R. McKeown, "Predicting the semantic orientation of adjectives", In: Proc. of the 35th Annual Meeting of the Association for Computational Linguistics and Eighth Conference of the European Chapter of the Association for Computational Linguistics, pp. 174-181, 1997.

[16] P.D. Turney and M.L. Littman, "Measuring praise and criticism: Inference of semantic orientation from association", ACM Transactions on Information Systems, Vol.21, No.4, pp.315-346, 2003.

[17] J. Kamps, M. Marx, R.J. Mokken, and M. De Rijke, "Using WordNet to Measure Semantic Orientations of Adjectives", In: Proc. of the 4th International Conference on Language Resources and Evaluation, pp. 1115-1118, 2004.

[18] S. Baccianella, A. Esuli, and F. Sebastiani, "Sentiwordnet 3.0: an enhanced lexical resource 
for sentiment analysis and opinion mining", In: Proc. of the 7th International Conference on Language Resources And Evaluation, pp. 22002204, 2010.

[19] K. Toutanova, D. Klein, C.D. Manning, and Y. Singer, "Feature-rich part-of-speech tagging with a cyclic dependency network", In: Proc. of the 2003 Conference of the North American Chapter of the Association for Computational Linguistics on Human Language Technology, pp. 173-180, 2003.

[20] R. Rada, H. Mili, E. Bicknell, and M. Blettner, "Development and application of a metric on semantic nets", IEEE Transactions on Systems, Man, and Cybernetics, Vol.19, No.1, pp.17-30, 1989.

[21] M.C. De Marneffe and C.D. Manning, "The Stanford typed dependencies representation", In: Proc. of the 22nd International Conference on Computational Linguistics, pp. 1-8, 2008.

[22] J. Lafferty, A. McCallum, and F.C. Pereira, "Conditional random fields: Probabilistic models for segmenting and labeling sequence data", In: Proc. of the Eighteenth International Conference on Machine Learning, pp. 282-289, 2001.

[23] C. Fellbaum, "WordNet", MIT Press, USA, 1998.

[24] E. van Miltenburg, "WordNet-based similarity metrics for adjectives", In: Proc. of the Eighth Global WordNet Conference, pp. 27-30, 2016. 